\title{
DESCRIPCIÓN DEL EMBARAZO ECTÓPICO EN EL HOSPITAL SAN JUAN DE DIOS DE CALI, COLOMBIA, ENTRE EL 2005 Y EL 2006
}

\section{Description of ectopic pregnancy at the Hospital San Juan de Dios de Cali, Colombia, 2005-2006}

\author{
Olga Lucía Cuero-Vidal, M.D.*
}

Recibido: septiembre 7/07 - Aceptado: noviembre 11/08

\section{RESUMEN}

Objetivos: calcular la razón de incidencia y describir las características sociodemográficas, clínicas y el tratamiento de las mujeres con embarazo ectópico (EE), que fueron atendidas en el Hospital San Juan de Dios de Cali (HSJD), entre el 1 de abril de 2005 y el 31 marzo de 2006.

Diseño del estudio: es un estudio descriptivo prospectivo, con información de la base de datos del departamento de estadística del HSJD y los datos de una encuesta estructurada para las mujeres con EE. Resultados: se atendieron 3722 nacidos vivos en este período y hubo 78 mujeres con EE. La incidencia fue del 2,1\%, el promedio y la mediana de edad fue 26 años, el 65,4\% no tenían ningún régimen de seguridad social, el 79\% eran remitidas de otras instituciones. El dolor abdominal fue la principal causa de consulta (42,3\%); el 53,8\% no planificaban, entre las que planificaban el 29\% lo hacían con oclusión tubárica bilateral, hubo un $7,7 \%$ con EE previo y éste era su segundo embarazo y su segundo ectópico. De las 78 mujeres con EE todas fueron llevadas a laparotomía y el promedio de hemoperitoneo fue $544 \mathrm{cc}$ y la mediana $500 \mathrm{cc}$, 59\% eran ectópicos derechos y el 88,5\% estaban

* Médica de la Universidad del Valle, especialista en Ginecología y Obstetricia de la Universidad de Antioquia, candidata al titulo de Magíster en Epidemiología de la Universidad del Valle. Ginecóloga Obstetra del Hospital San Juan de Dios y Docente de la Facultad de Medicina de la Universidad Santiago de Cali. Cali, Colombia. Correo electrónico: linary67@hotmail.com rotos, en el 96,7\% de los casos el tratamiento fue salpingectomía total.

Conclusión: la incidencia de embarazo ectópico fue del 2,1\%; considerada como alta con respecto a la reportada en la literatura, predomina el diagnóstico tardío de la entidad en esta comunidad, lo cual no permitió tratamientos conservadores.

Palabras clave: embarazo ectópico, incidencia, diagnóstico, tratamiento.

\section{SUMMARY}

Objectives: collecting data regarding the incidence of ectopic pregnancy and determining baseline and clinical characteristics and the treatment provided for women with ectopic pregnancy who were attended at Hospital San Juan de Dios de Cali (HSJD) between 1st April 2005 and 31st March 2006.

Materials and methods: this was a descriptive, prospective study using data obtained from the HSJD statistics department's data system and a structured survey of women with ectopic pregnancy.

Results: there were 3,722 live births during this period; 78 were diagnosed as ectopic pregnancy (2,1\% incidence, 26 mean and median age). 65,4\% had no social security and $79 \%$ arrived from other institutions. Abdominal pain was the main reason for consulting (42,3\%). 53,8\% did not use family-planning methods; $29 \%$ of those did engage in family-planning used bilateral tubal occlusion. 7,7\% had had a prior ectopic pregnancy; 3 of these cases were having their second pregnancy and second ectopic 
pregnancy. All 78 women with ectopic pregnancy were sent for laparatomy. Mean haemoperitoneum was $544 \mathrm{cc}$ and median 500cc. 59\% had right ectopic pregnancy and $88,5 \%$ were suffering from ectopic rupture. Total salpingectomy was the treatment provided in $96,7 \%$ of the cases.

Conclusion: ectopic pregnancy incidence was $2,1 \%$, this being considered high when compared to that reported in the literature. Late diagnosis of ectopic pregnancy in this community predominated, thereby precluding conservative treatment.

Key words: ectopic pregnancy, incidence, diagnosis, treatment.

\section{INTRODUCCIÓN}

El embarazo ectópico (EE) es la implantación del óvulo fecundado en cualquier parte de la cavidad pelviana o abdominal que no sea la cavidad uterina, ${ }^{1-6}$ entre sus principales complicaciones se describe la muerte por hemorragia peritoneal y la disminución o pérdida de la fertilidad. ${ }^{1-7}$

La incidencia de embarazo ectópico ha ido aumentando en las últimas décadas ${ }^{1,5,6}$ debido a un incremento en el número de infecciones de transmisión sexual, ${ }^{6-8}$ inducción de la ovulación, ${ }^{5,9}$ el aborto inducido, ${ }^{1,10}$ la cirugía reparadora de la trompas, ${ }^{1,10,11}$ la presencia de un embarazo ectópico previo, ${ }^{1,11}$ las técnicas de fertilización asistida ${ }^{11}$ y el uso de algunos tipos de anticoncepción. ${ }^{10-12}$

Las incidencias de EE, según el número de nacidos vivos, se reportan en Estados Unidos del 1,8 al $2 \% ;{ }^{13}$ en Inglaterra el $1,6 \%{ }^{14}$ y en Guinea el $1,5 \% ;{ }^{15}$ en Colombia se conoce que es la cuarta causa de hospitalización en el Hospital de Caldas en Manizales. ${ }^{16}$

Aunque el embarazo ectópico sigue siendo la principal causa de mortalidad materna en el primer trimestre en países industrializados, ${ }^{1,4,5}$ el avance en las tecnologías para diagnóstico temprano ha llevado a una disminución drástica de la mortalidad por embarazo ectópico, casi en un $90 \% .{ }^{1,2,4,5,17}$ Esto ha sido posible gracias a la disponibilidad de la Beta Gonadotropina Coriónica ( $\beta \mathrm{hCG}$ ), la laparoscopia, los niveles de progesterona ${ }^{17}$ y la ecografía transvaginal (ETV). ${ }^{18}$
El diagnóstico temprano del EE no roto permite el uso de tratamientos conservadores que preservan las trompas de Falopio, ${ }^{19}$ dentro de los cuales se encuentra el manejo expectante, ${ }^{20} \mathrm{el}$ manejo médico usando metotrexato ${ }^{20-22}$ o la cirugía conservadora, ya sea salpingostomía, salpingectomía parcial o la expresión de la fimbria. ${ }^{23}$

El Hospital San Juan de Dios de Cali es un nivel II de atención, es un sitio de referencia para el diagnóstico y manejo del embarazo ectópico. Se desconoce la incidencia de esta patología en este hospital, las características clínicas y paraclínicas de las pacientes que allí ingresan, la manera como se hace el diagnóstico y cuáles son las alternativas terapéuticas que se utilizan. Por tal motivo, se decidió realizar un estudio para describir el comportamiento del embarazo ectópico en dicha institución entre abril de 2005 y abril de 2006.

\section{MATERIALES Y MÉTODOS}

Se diseñó un estudio cohorte descriptivo y se tomó como población de estudio el universo de las mujeres atendidas en el servicio de obstetricia y ginecología en el Hospital San Juan de Dios de Cali, entre el 1 de abril de 2005 y el 31 de marzo de 2006 con diagnóstico de embarazo ectópico. Este hospital es uno de los centros de atención de pacientes obstétricas con patología nivel II, atiende la población de bajo y medio nivel socioeconómico de la ciudad de Cali y municipios aledaños del sur occidente colombiano.

Entre los criterios de inclusión estaban mujeres en edad reproductiva, cuyo diagnóstico al ingreso fuera embarazo ectópico. La fuente de información fue una encuesta estructurada aplicada por el investigador a las pacientes, donde se consignaron sus datos demográficos, la presencia de factores de riesgo, se revisaron sus historias clínicas y se completaron los datos con la información dada por el médico tratante. Para el cálculo de la razón de incidencia de embarazo ectópico en el HSJD durante este período, se utilizaron los registros de estadística del HSJD.

Variables a medir: datos demográficos como edad, seguridad social, estado civil, escolaridad, 
etnia y ocupación; la presencia de factores de riesgo descritos en la literatura, las condiciones clínicas y paraclínicas de ingreso, los criterios diagnósticos, los hallazgos operatorios, las complicaciones como choque hipovolémico, pérdida de la fertilidad, infección posoperatoria o muerte.

Los datos fueron recopilados y analizados en Epi Info 3.2.2, para las variables continuas se utilizaron los rangos, medias y medianas, y las variables categóricas se registraron en porcentajes.

Se determinó la razón de incidencia como el número de mujeres con embarazo ectópico en relación al número de nacidos vivos durante el período de estudio en la institución.

\section{RESULTADOS}

Entre el 1 de abril de 2005 y el 31 de marzo de 2006 se atendieron en HSJD un total de 3722 nacidos vivos, durante este tiempo hubo 78 mujeres con embarazo ectópico confirmado por patología, obteniéndose una razón de incidencia de EE de 2,1\% (78/3722) en este período.

La edad de las pacientes osciló entre 14 y 38 años, con un promedio y una mediana de 26 años. El 11,5\% eran adolescentes (entre 10 y 19 años de edad según OMS), el 83,4\% (65) tenían entre 20 y 34 años. Los datos sobre estado civil, ocupación, escolaridad, seguridad social, causa de consulta, de remisión y procedencia se reportan en la tabla 1 y la descripción de los factores de riesgo se registra en la tabla 2.

\begin{tabular}{|c|c|c|c|}
\hline Variable & No (\%) & Variable & No (\%) \\
\hline Estado civil & & Seguridad social & \\
\hline Soltera & $28(35,9)$ & Ninguna & $65,4(51)$ \\
\hline UL & $46(59)$ & ARS & $26,9(21)$ \\
\hline Casada & $2(2,6)$ & EPS & $7,7(6)$ \\
\hline Separada & $1(1,3)$ & & \\
\hline \multicolumn{4}{|l|}{ Ocupación } \\
\hline Hogar & $27(34,6)$ & Causa de consulta & \\
\hline Desempleada & $7(9)$ & Dolor & $33(42,3)$ \\
\hline Estudiante & $6(7,7)$ & Sangrado y dolor & $24(30,8)$ \\
\hline Oficios varios & $4(5,2)$ & Sangrado, dolor, amenorrea & $10(12,8)$ \\
\hline Independiente & $4(5,1)$ & Sangrado & $9(11,5)$ \\
\hline Otros & $5(6,4)$ & Amenorrea & $2(2,6)$ \\
\hline \multicolumn{4}{|l|}{ Etnia } \\
\hline Negra & $19(24,3)$ & Remisión & \\
\hline Mestiza & $18(23,1)$ & Sí & $62(79)$ \\
\hline Blanca & $17(21,8)$ & No & $16(21)$ \\
\hline India & $2(2,6)$ & & \\
\hline Sin dato & $22(28,2)$ & Causa remisión & \\
\hline Escolaridad & & Ectópico & $42(67,7)$ \\
\hline Primaria & $13(16,7)$ & Aborto & $6(9,7)$ \\
\hline Básica secundaria & $19(24,6)$ & Dolor abdominal & $6(9,7)$ \\
\hline Bachillerato completo & $8(10,3)$ & Apendicitis & $4(6,5)$ \\
\hline Tecnología & $4(5,2)$ & EPI & $3(4,8)$ \\
\hline Sin dato & $34(43,6)$ & Otras & $2(2,5)$ \\
\hline
\end{tabular}




\begin{tabular}{|l|l|}
$\begin{array}{l}\text { Tabla 2. Presencia de factores de riesgo } \\
\text { en mujeres con embarazo ectópico, }\end{array}$ \\
Hospital San Juan de Dios Cali - Colombia \\
2005-2006.
\end{tabular}

Entre las 78 mujeres con EE, el 7,7\% (6) cursaban su segundo ectópico, 3 de ellas no habían tenido antes ningún embarazo intrauterino (era su segundo embarazo) y en el embarazo ectópico previo habían sido llevadas a salpingectomía total. De las 6 pacientes que cursaban su segundo embarazo ectópico, ninguna reunía criterios para un manejo conservador y todas fueron llevadas a salpingectomía total. Los datos sobre las características clínicas y paraclínicas al ingreso, el método diagnóstico y el tratamiento, se describen en la tabla 3.

El tiempo transcurrido entre el ingreso y el procedimiento quirúrgico fue entre 25 minutos y 4 días, con un promedio de 18,5 horas, la mediana fue 11,5 horas, y el 53,5\% (38) se llevaron a cirugía en menos de 12 horas. Hubo 3 pacientes que eran RH negativas, ninguna recibió inmunoglobulina

\section{Tabla 3. Características clínicas, paraclínicas, diagnóstico y tratamiento de embarazo ectópico en Hospital San Juan de Dios Cali - Colombia 2005-2006.

\begin{tabular}{|c|c|}
\hline Variable & No (\%) \\
\hline Estado al ingreso & \\
\hline
\end{tabular}

\begin{tabular}{l|c|}
\hline TA Normal* & $69(88,5)$ \\
\hline Hipotensión & $4(11,5)$ \\
\hline Luce pálida & $30(35)$ \\
\hline Dolor abdominal & $50(64)$ \\
\hline Abdomen agudo & $21(27)$ \\
\hline Diagnóstico & \\
\hline Prueba de embarazo & \\
\hline Positiva* & $65(83.3)$ \\
\hline Negativa & $1(1,3)$ \\
\hline No se hizo & $12(15,4)$ \\
\hline Ecografía & \\
\hline No se realizó & $12(15,4)$ \\
\hline Se realizó*** & $84,6(66)$ \\
\hline Hallazgos y tratamiento & \\
\hline Localización ectópico & \\
\hline Derecho & $59(46)$ \\
\hline Izquierdo & $41(32$ \\
\hline Ectópico roto & $88,5(69)$ \\
\hline Ectópico no roto & $11,5(9)$ \\
\hline Salpingectomía total & $96,7(75)$ \\
\hline Salpingectomía parcial & $3,3((3)$ \\
\hline Planificación al alta & $30,8(24)$ \\
\hline$* T A:$ Tensión arterial & \\
\hline$* *$ Se le realizó $\beta$ hCG cuantitativa 21 el $32,3 \%$ \\
\hline$* * 3(4,5 \%)$ Ecografías reportadas normales \\
\hline
\end{tabular}

anti-D. Todas la pacientes fueron llevadas a laparotomía, el hemoperitoneo encontrado osciló entre 0 y $2500 \mathrm{cc}$, con un promedio de $554 \mathrm{cc}$ y una mediana de 400cc, y en el 32\% (25) se encontró un hemoperitoneo mayor de 500cc. No se presentó ningún evento de mortalidad.

\section{DISCUSIÓN}

En la mayoría de Europa y Norte América la incidencia de EE se ha triplicado en los últimos 30 años 
y se estima en un 2\% de los nacidos vivos; en 1975 se consideraba que era menor del 1\%. ${ }^{1-5,8,13-15}$ En este estudio, la incidencia de EE hallada en el HSJD de Cali es similar a la reportada en la literatura, la cual se considera alta. Cabe resaltar que éste es un centro de remisión nivel II, donde se atiende pacientes de estratos 1, 2 y 3 principalmente, lo cual indica que no representa la incidencia de la enfermedad en la población general de Cali.

Se atribuyen como causas principales de esa mayor incidencia, la epidemia de enfermedades de transmisión sexual, ciertas intervenciones médicas, el aborto inducido, la cirugía reparadora de la trompa o algunas circunstancias que se dan en la mujer, como la tendencia a retrasar el momento de tener hijos, la presentación anterior de otro EE o la infertilidad..$^{1-5,7}$ En este estudio, de las mujeres en las que se pudo obtener estos datos, más del $40 \%$ no presentan ningún factor de riesgo.

Los estudios describen que sólo en el 50\% de los casos es posible hacer el diagnóstico por clínica y las mujeres con EE generalmente consultan por dolor abdominal tipo cólico, en el 90\% de los casos intermitente, o dolor súbito agudo asociado con frecuencia a lipotimia o síncope, la amenorrea es la segunda causa de consulta. ${ }^{1-6}$ En nuestro estudio el dolor fue la principal causa de consulta, pero no superó el 43\%, y la amenorrea fue la última. Adicionalmente, en el momento del diagnóstico se deben tener en cuenta los diagnósticos diferenciales como EPI, apendicitis, cuerpo lúteo roto, torsión de la trompa, litiasis renal, entre otros, siendo los dos primeros los más frecuentes. ${ }^{1-6}$ En este estudio los diagnósticos diferenciales más frecuentes, como EPI y apendicitis, fueron las dos últimas causas de remisión.

El EE es una entidad clínica que genera dificultades en el diagnóstico y simula múltiples patologías, si el médico no considera el EE entre su diagnóstico en mujeres en edad reproductiva, puede retrasar su oportuno manejo. ${ }^{1-6}$ Es por eso que la combinación de los niveles de $\beta$ hCG y la ETV, ha hecho posible el diagnóstico temprano del EE, ${ }^{17,18}$ haciendo que las pacientes sean susceptibles de un tratamiento conservador, que preserve las trompas de Falopio e incluso la cirugía puede ser reemplazada por manejo médico ${ }^{19-25}$, en nuestro estudio la combinación de estos métodos fue poco utilizada.

Se describe que en la actualidad se rompen el 26\% de los ectópicos y hasta un 10\% pueden presentar choque hipovolémico. ${ }^{1,47}$ En este estudio el EE roto y el choque hipovolémico superaron el 88 y el $11 \%$ respectivamente, predominando el diagnóstico tardío de la enfermedad en esta comunidad. Una posible hipótesis para este retraso en el diagnóstico, podría ser la falta de afiliación al régimen de seguridad social, lo cual genera una barrera de acceso a los servicios de salud, que en este estudio superó el 65\%; por tal razón sería importante evaluar este factor.

Adicionalmente 3 de nuestras pacientes, a pesar de tener como factor de riesgo el antecedente de un embarazo ectópico previo y no tener otras gestaciones, terminaron en salpingectomía total bilateral, perdiendo su fertilidad, y para lograr tener un hijo tendrán que recurrir a técnicas de fertilización asistida. Es importante anotar que estas últimas no están cubiertas por ningún régimen de seguridad social.

Se describe que la localización más frecuente fue el lado derecho, encontrada entre un 50 y 69\%, ${ }^{11}$ lo cual coincide con los hallazgos de nuestro estudio.

Las limitaciones están dadas por la dificultad en obtener los datos completos por historias clínicas y las dificultades cuando se registra el código CIE - 10 de EE, no se realizó una evaluación económica de los gastos que implica a las instituciones el manejo de pacientes con diagnósticos tardíos de EE.

\section{CONCLUSIONES}

En este estudio la incidencia de EE en el HJSD es muy similar a la reportada en otros estudios, predominando el diagnóstico tardío y los procedimientos radicales que afectan la fertilidad en dichas mujeres. Se sugiere otros diseños epidemiológicos que eva- 
lúen el impacto económico y determinen cuáles son lo factores asociados al diagnóstico tardío del EE.

\section{AGRADECIMIENTOS}

A los internos de la Universidad Santiago de Cali: Alejandro Moncayo, Luis Fernando Román, Alberto García y a los estudiantes de Medicina.

Al Director del Hospital San Juan de Dios: Doctor Iván González y al personal de estadística del hospital por su colaboración.

\section{REFERENCIAS}

1. Cunningham FG, Gant NF, Leveno KJ, Gilstrap LC, Haunt JC, Wenstrom KD. Embarazo ectópico. En: Williams Obstetricia. 21 ed. 2001. p. 757-79.

2. Pisarska MD, Carson SA. Embarazo ectópico. En: Tratado de Obstetricia y Ginecología. 8a . ed. 2000. p. 161-79.

3. Casanova C. Epidemiología del embarazo ectópico. Revista Cubana de Enfermería 2003;19(3).

4. Solovera S. Embarazo ectópico. Revisión bibliográfica 1992-2003. Visitado 2007 Ago 30. Disponible en: http://www.cedip.cl/documentos/archivos/33.html

5. Herranz G. Epidemiología del embarazo ectópico; 1990. Visitado 2007 Ago 30. Disponible en: http:// apuntesanatomia.iespana.es/hip/embarazo.htm

6. Ruiz AI, Ñañez H. Embarazo ectópico. En: Texto de Obstetricia y Perinatología. Bogotá-Colombia: Universidad Nacional de Colombia-Instituto Materno Infantil; 1999. p. 233-50.

7. Birkhahn R, Gaeta T, Van Deusen S, Tloczkowski J. The ability of traditional vital signs and shock index to identify ruptured ectopic pregnancy. Am J Obstet Gynecol 2003;189:1293-6.

8. Hill G, Hebert CM. Embarazo ectópico. En: Copeland LJ, Jarell LF, Mogregor J, editors. Ginecología. 1a. ed; 1994. p. 237-53.

9. Balestena JM, Rodríguez A, Reguera O. Factores epidemiológicos del embarazo ectópico. Rev Cubana Obstet Ginecol 2002;28(3). Visitado 2008 Oct 05. Disponible en: http://scielo.sld.cu/scielo. php? script $=$ sci_arttext\&pid $=$ S0138-600X200200 0300004\&lng $=\mathrm{es} \& n r m=\mathrm{iso \& t} \operatorname{lng}=\mathrm{es}$

10. Bouyer J, Coste J, Shojaei T, Pouly JL, FernándezH, Gervaud L, et al. Risk factors for ectopic pregnancy: a comprehensive analysis based on a large case-control, population-based study in France. Am J Epidemiol 2003;157:185-94.

11. Luglio JM, Díaz L, Cáceres E. Características clínicopatológicas de las pacientes con diagnóstico de embarazo ectópico en el Hospital Nacional Edgardo Rebagliati Martins entre 1998 y 2002. Rev Med Hered 2005;16:26-30.

12. González L, Cabrera C, Jiménez J, Grössl J. Embarazo ectópico ovárico con dispositivo intrauterino in situ: caso clínico. Rev Obstet Ginecol Venez 2005;65:203-6.

13. Saraiya M, Berg CJ, Shulman H, Green CA, Atrash HK. Estimates of the annual number of clinically recognized pregnancies in the United States, 1981-91. Am J Epidemiol 1999;149:1025-9.

14. Raikhowa M, Glass MR. Rutherford AJ, Balen AH, Sharma V. Cuckle HS. Trend in the incidence of ectopic pregnancy in England and Wales from 1966 to 1996. BJOG 2000;107:369-74.

15. Thonneau P, Hijazi Y, Goyaux N, Calvez T, Keila N. Ectopic pregnancy in Conakry, Guinea. Bull World Health Organ 2002;80:365-70.

16. Jaramillo E. Morbi-mortalidad materna en el Hospital de Caldas, Manizales. Colombia Med 1997;28:77-84.

17. Enríquez B, Fuentes L, Gutiérrez N, León M. Tendencias actuales en el tratamiento del embarazo ectópico. Rev Cubana Obstet Ginecol 2003;29(3). Visitado 2008 Oct 05. Disponible en: http://scielo. sld.cu/scielo.php?script $=$ sci_arttext\&pid $=$ S0138$600 \times 2003000300002 \& \operatorname{lng}=\mathrm{es} \& \mathrm{nrm}=\mathrm{iso \& t} \operatorname{lng}=\mathrm{es}$

18. Levine D. Ectopic pregnancy. En: Callen PW. Ultrasonography in Obstetrics and Gynecology. 4 ed. 2000. p. 912-34.

19. Lipscomb G, Stovall T, Ling F. Nonsurgical treatement of ectopic pregnancy. N Engl J Med 2004;343:1325-9.

20. Teran J. Metotrexate y conducta expectante en el embarazo ectópico no roto. Rev Obstet Ginecol Venez 2002;62:161-74.

21. Nelson AL, Adams Y, Nelson LE, Lahue AK. Ambulatory diagnosis, and medical management of ectopic pregnancy in public teaching hospital serving indigent women. Am J Obstet Gynecol 2003;188:1541-50.

22. Stovall T. Medical management should be routinely used as primary therapy for ectopic pregnancy. Clin Obstet Gynecol 1995;38:347-61.

23. Zarhi J, Campana C, Brito R, Stuardo P, Schalper A. Manejo laparoscópico conservador de embarazo cornual. Rev Chil Obstet Ginecol 2003;68:36-41. 